Rev. Elev. Méd. vét. Pays trop., 1973, 26 (2) : 151-67

\title{
Revue des connaissances actuelles sur la cytogénétique des Glossines (Diptera)
}

\author{
par J. ITARD $(*)$
}

\begin{abstract}
RESUME
Les caryotypes de douze espèces de Glossines ont été décrits par différents auteurs, au cours des six dernières années. Toutefois, parmi celles-ci, sept espèces seulement ont été relativement bien étudiées : deux espèces du groupe palpalis ( $G$. fuscipes fuscipes et $G$, tachinoides), quatre espèces du groupe morsitans (G.m. morsitans, G.m. centralis, G.m. sub. morsitans et $G$. austeni) et une espèce du groupe fusca (G. fusca congolensis).

La carte des chromosomes polyteniques a pu, en outre, être établie chez une espèce (G.m. morsitans).
\end{abstract}

\section{INTRODUCTION}

La lutte génétique, qui constitue la méthode la plus moderne de lutte contre les insectes nuisibles, suppose une connaissance approfondie de la génétique fondamentale de ces insectes.

Une mise au point des acquisitions effectuées dans ce domaine a été publiée par l'O.M.S. en 1967, dans le volume intitulé * Genetics of insect vectors of disease $»(19)$.

La lecture de cet important ouvrage montre que si des renseignements précieux ont été obtenus chez certains insectes nuisibles, tels que Aedes aegypti, Culex pipiens, plusieurs Anopheles, Musca domestica et divers diptères Mucoïdes, certains acariens, des triatomes, les blattes, de nombreuses autres espèces vectrices, dont les mouches tsé-tsé, pour lesquelles les renseignements étaient inexistants ou fragmentaires, ne sont pas mentionnées.

En effet, le genre Glossina, vecteur des trypanosomoses africaines humaines et animales, n'avait fait, jusqu'à ces dernières années, l'objet

(*) I.E.M.V.T., Service Entomologie, 10 rue Pierre Curie, 94700 Maisons-Alfort, France. que d'une seule observation sur les chromosomes de $G$. morsitans [SLYSINSKI, in VANDERPLANK (18)].

Ce n'est qu'à partir de 1966 que des études plus complètes ont été publiées. Nos connaissances sur la génétique fondamentale des mouches tsé-tsé, bien qu'encore très incomplètes, ont alors progressé.

Il n'est pas dans notre propos de faire une étude exhaustive de la génétique des glossines. Notre seul but est de rassembler dans ce document les renseignements obtenus par divers auteurs et de faire apparaître plus clairement les lacunes existantes, dans l'espoir de susciter ainsi de nouvelles recherches.

\section{ETAT DES CONNAISSANCES ACTUELLES}

A l'exception de l'observation, non publiée, de SLYSINSKI, la première description des chromosomes de Glossines a paru en 1966.

Elle concerne $G$. tachinoides West., 1850, qui possède $2 \mathrm{n}=6$ chromosomes somatiques et $G$. morsitans morsitans West., 1850; MACHADO, 1970 (ex- G. morsitans orientalis 
Vand., 1949), qui possède $2 \mathrm{n}=10$ chromosomes somatiques (ITARD, 5).

Dans les années suivantes furent décrits des caryotypes de :

- G. palpalis palpalis R.D., 1830 (2 $\mathrm{n}=$ 6 chromosomes) (RIORDAN, 13).

- G. fuscipes fuscipes New, 1910 (2 n $=$ 6 chromosomes) (MAUDLIN, 10 et 11 ; ITARD, 7).

- G. longipalpis Wied., 1830 ( $2 \mathrm{n}=8$ chromosomes) (BALDRY, 1).

- G. pallidipes Austen, 1903 ( $2 \mathrm{n}=8$ chromosomes) (HULLEY, 4; MAUDLIN, 11).

- G. morsitans centralis MACHADO, 1970 ( $G$. morsitans morsitans West., 1850) (2 n $=8$ chromosomes) (MAUDLIN, 11).

- G. swynnertoni Austen, 1923 (2 $\mathbf{n}=$ 8 chromosomes) (MAUDLIN, 11).

- G. austeni New., 1912 ( $2 \mathrm{n}=14$ chromosomes) (ITARD, 7).

- G. brevipalpis New., 1910 ( $2 \mathrm{n}=16$ chromosomes) (MAUDLIN, 11).

- G. fusca congolensis New. et EVANS, 1921 (2 $\mathrm{n}=22$ chromosomes) (ITARD, 9).

Toutefois ces descriptions sont, dans la plupart des cas, incomplètes, car les techniques utilisées jusqu'alors n'ont généralement pas permis de différencier les chromosomes sexuels des autosomes.

L'étude du développement de l'appareil génital mâle chez la pupe de glossine (ITARD, 8) a permis de préciser le moment où se produit, dans les cellules sexuelles, la division méiotique. La dissection de pupes âgées de 6 à 10 jours, suivant l'espèce, et la coloration du ganglion nerveux péri-œsophagien chez la pupe femelle, du ganglion nerveux et des testicules chez la pupe mâle, permettent ainsi de dresser le caryotype des deux sexes.

SOUTHERN et ses collaborateurs ont également étudié le déroulement de la méiose chez G. morsitans morsitans (15), et ont ainsi précisé certains aspects de la séquence méiotique chez cette espèce. Ils ont en outre, tout récemment, complété ces recherches par une étude comparative des caryotypes de quatre espèces du sous-genre Glossina (groupe morsitans), à savoir G.m. morsitans, G.m. submorsitans, G.m. centralis, G. austeni $(12,16)$, ainsi que par l'étude ultrastructurale des chromosomes en méiose chez G. austeni et G.m. morsitans (3).

Enfin les chromosomes polyténiques ont été mis en évidence chez $G$. palpalis palpalts (BURCHARD et BALDRY, 2; RIORDAN, 14), mais ces auteurs n'ont pu dresser la carte de ces chromosomes géants. Par contre, SOUTHERN et ses collaborateurs ont réussi dernièrement à dresser la carte des chromosomes polyténiques chez G.m. morsitans (17).

\section{TECHNIQUES D'ETUDES}

\section{Matériel}

Seul SLYSINSKI a pu mettre en évidence des chromosomes chez la Glossine adulte. Tous les auteurs qui ont, par la suite, recherché des chromosomes chez l'imago, ont abouti à un échec.

La recherche des cellules en mitose se fait chez la larve au dernier stade, ou plus commodément chez la pupe âgée de quelques jours, dans le ganglion nerveux péri-œsophagien.

Les chromosomes en méiose sont régulièrement trouvés dans les testicules de la pupe mâle âgée de 6 à 10 jours.

Les chromosomes polyténiques ont été décelés dans le tissu musculaire de pupes âgées de 36 à 48 heures (RIORDAN, 14), ou dans les cellules trichogènes et tormogènes des macrochètes thoraciques chez la pupe âgée de 17 jours (SOUTHERN, 17).

\section{Méthode de dissection}

La technique d'isolement des tissus est relativement simple. Les larves ou les pupes sont disséquées sous la loupe binoculaire, dans quelques gouttes de sérum physiologique. L'enveloppe pupale est fendue au bistouri, dans le sens longitudinal et le contenu interne de la pupe isolé sur une lame, dans une ou deux gouttes de sérum physiologique. A l'aide de fines aiguilles, on isole les organes désirés qui sont transférés sur des lames propres en vue de leur fixation et de leur coloration.

SOUTHERN, pour la recherche des chromosomes en méiose, ajoute une goutte d'eau distillée, avant coloration des testicules, pendant 1 ou 2 minutes, pour gonfler le cytoplasme. 


\section{Fixation}

La fixation n'est pas toujours nécessaire. Certains auteurs (BALDRY, RIORDAN) fixent les tissus, pendant quelques minutes, dans une solution à parties égales d'acide acétique à 45 p. 100 et d'alcool éthylique à 95 p. 100. HULLEY utilise l'alcool propionique, ce qui lui permet de conserver les tissus pendant près d'un mois avant de les colorer.

La plupart des autres auteurs omettent ce premier temps, les colorants utilisés agissant également comme fixateur.

\section{Coloration}

Le colorant de base est l'orcéine, en solution dans de l'acide acétique, de l'acide lactique, de l'acide propionique, ou un mélange de deux de ces acides. Personnellement, après avoir utilisé une solution d'orcéine à 2 p. 100 dans un mélange à parties égales d'eau, d'acide lactique et d'acide acétique, nous avons, comme HULLEY, remplacé l'acide acétique par de l'acide propionique. SOUTHERN utilise une solution à 5 ou 8 p. 100 d'orcéine dans de l'acide acétique à 45 p. 100 . BALDRY et RIORDAN ont employé une solution à 2 p. 100 d'orcéine dans un mélange à parties égales d'acide acétique glacial et d'acide lactique à 85 p. 100.

Les temps de coloration sont très variables suivant les différents auteurs. HULLEY laisse les tissus dans le colorant pendant toute une nuit. RIORDAN réduit le temps de coloration à 8 heures et BALDRY à 10 minutes. SOUTHERN colore pendant une heure après avoir, au bout d'une minute, ajouté au colorant la moitié de son volume d'acide acétique à $45 \mathrm{p}$. 100.

Les temps que nous avons adoptés dans notre laboratoire varient, suivant l'intensité de coloration désirée, de 1 heure à 3 heures.

Dans tous les cas, il est prudent de recouvrir la préparation avec un couvercle de boîte de Pétri pour limiter l'évaporation.

Quelques auteurs, dont nous-mêmes, rincent ensuite la préparation à l'acide acétique pour éliminer l'excès de colorant. Ce temps est négligé par d'autres auteurs, dont SOUTHERN, qui chauffe légèrement la lame à la fin du temps de coloration. RIORDAN, pour la préparation des chromosomes polyténiques, recommande d'effectuer toutes les opérations, depuis la dissection jusqu'à la fin de la coloration, dans une chambre froide à $4^{*} \mathrm{C}$. SOUTHERN, pour la mise en évidence de ces mêmes chromosomes, hydrolyse les tissus dans l' $\mathrm{HCl} 5 \mathrm{~N}$ pendant 30 minutes à $22^{\circ} \mathrm{C}$, puis colore au Feulgen pendant 2 heures.

\section{Montage et examen}

Aussitôt après la coloration (ou le rinçage à l'acide acétique), une lamelle est délicatement déposée sur la préparation, qui est écrasée, entre deux couches de papier filtre, par pression du pouce. La pression doit être assez forte pour bien étaler la préparation et obtenir une couche monocellulaire. Il faut veiller à ce que la lamelle ne soit pas déplacée, faute de quoi la préparation serait illisible. Celle-ci est ensuite scellée au vernis à ongle incolore et peut être alors conservée un mois au réfrigérateur.

L'examen s'effectue au microscope à contraste de phase, à sec puis à l'immersion. Les chromosomes apparaissent ainsi nettement et peuvent être aisément photographiés.

\section{Différenciation des chromosomes sexuels}

L'identification des hétérosomes s'effectue en comparant les chromosomes en métaphase dans les cellules somatiques des pupes mâles avec ceux des pupes femelles. Cet examen permet d'associer chaque chromosome avec son homologue et d'établir ainsi des paires, sauf dans l'un des sexes (le mâle en l'occurence) chez qui on trouve deux chromosomes morphologiquement différents et ne pouvant être associés avec aucun des autres chromosomes de la même cellule. L'un de ceux-ci est identique aux chromosomes de l'une des paires du sexe opposé. Il s'agit du chromosome X. Le chromosome restant est le chromosome $\mathrm{Y}$.

L'étude du jeu haploïde des chromosomes en méiose dans les cellules sexuelles de la pupe mâle permet de confirmer cette identification. Le chromosome $\mathbf{X}$ est, comme les grands autosomes, euchromatique, alors que le chromosome $\mathrm{Y}$ est hétérochromatique. A la prophase méiotique, les grands autosomes et le chromosome $\mathrm{X}$ apparaissent les premiers (fig. 30). Pendant la prophase I, les grands autosomes se condensent et s'éloignent du chromosome X. Le chromo- 
some $\mathrm{Y}$ apparaît alors, réuni au chromosome $\mathrm{X}$ par un court segment, près du centromère.

\section{CARYOTYPES DES DIFFERENTES ESPECES DE GLOSSINES}

Les systématiciens divisent le genre Glossina en trois sous-genres : s.g. Nemorhina, s.g. Glossina, s.g. Austenina, qui correspondent respectivement aux anciens groupes palpalis, morsitans et fusca. L'étude des caryotypes paraissant confirmer cette division, nous étudierons successivement les espèces appartenant à chacun de ces sous-genres.

\section{Sous-genre Nemorhina (groupe palpalis)}

Les espèces appartenant à ce sous-genre, dont les caryotypes ont été décrits, sont :

$G$. tachinoides, $G$. fuscipes fuscipes, et $G$. palpalis palpalis.

Toutes ces espèces possèdent six chromosomes répartis en trois paires, que nous distinguerons, suivant la terminologie utilisée par SOUTHERN, en deux paires d'autosomes $L_{1}$ et $\mathrm{L}_{2}$ et deux chromosomes sexuels. Ces dernjers n'ont pas été différenciés des autosomes par RIORDAN et MAUDLIN, chez $G$. palpalis palpalis et chez $G$. fuscipes fuscipes. Cependant l'examen des caryotypes de $\boldsymbol{G}$. tachinoides et de $G$. fuscipes fuscipes, que nous avons récemment repris, nous ayant permis de distinguer les chromosomes sexuels des autosomes dans ces deux espèces, il nous paraît évident que toutes les espèces étudiées de ce groupe possèdent une formule chromosomique identique, à savoir :

\section{$2 \mathrm{n}=4$ autosomes +2 hétérosomes}

a) Glossina fuscipes fuscipes Newstead, 1910

La morphologie des chromosomes de cette espèce est illustrée dans les figures 1 à 9.

Les deux autosomes $\mathrm{L}_{t}$ sont les plus longs; ils sont sub-métacentriques, c'est-à-dire que la position du centromère divise chaque chromosome en deux bras de longueurs inégales, le bras le plus court représentant environ le $1 / 3$ de la longueur totale du chromosome. On remarque en outre une constriction secondaire sur le bras le plus long (fig. 2 et 3 ).

Les autosomes $\mathrm{L}_{2}$ sont nettement plus courts que les $\mathrm{L}_{1}$ (environ $1 / 3$ plus court); ils sont presque métacentriques, l'un des bras étant très légèrement plus court que l'autre. Enfin, ils ne présentent pas de constriction secondaire.

Les hétérosomes se différencient en un chromosome $\mathrm{X}$ et un chromosome $\mathrm{Y}$. La femelle est homogamétique et possède deux chromosomes X (fig. 1). Le mâle est hétérogamétique et possède un chromosome $\mathrm{X}$ et un chromosome Y (fig. 2 et 3 ).

Le chromosome $\mathrm{X}$ est sensiblement de la même longueur que l'autosome $\mathrm{L}_{1}$, mais il est nettement métacentrique (deux bras de longueurs égales) et ne possède pas de constriction secondaire.

Le chromosome $Y$ est plus court que l'autosome $\mathrm{L}_{1}$, mais plus long que l'autosome $\mathrm{L}_{2}$. Il est très nettement hétérobrachial, le bras le plus court ne faisant environ que le $1 / 5$ de la longueur totale du chromosome. Il ne possède pas de constriction secondaire.

b) Glossina tachinoides Westwood, 1850

Les figures 10 à 15 illustrent le caryotype de cette espèce.

Les autosomes comprennent, comme chez G. fuscipes fuscipes :

- une grande paire $\left(\mathrm{L}_{1}\right)$ submétacentrique, avec une constriction secondaire sur le bras le plus long (fig. 10 et 11).

- une paire moyenne $\left(\mathrm{L}_{2}\right)$ dont les deux bras sont presque égaux, sans constriction secondaire.

Le femelle possède deux chromosomes $\mathrm{X}$ et le mâle un chromosome $\mathrm{X}$ et un chromosome $Y$.

Les dimensions du chromosome $\mathbf{X}$ sont intermédiaires entre celles des autosomes $\mathrm{L}_{1}$ et $\mathrm{L}_{2}$. Il n'y a pas de constriction secondaire.

Le chromosome $\mathrm{Y}$ est le plus petit des chromosomes. Comme le chromosome $\mathrm{X}$, ses deux bras sont sensiblement égaux, à la différence du chromosome $Y$ de $G$. fuscipes fuscipes (fig. 12 et 15).

c) Glossina palpalis palpalis Robineau-Desvoidy, 1830.

Les chromosomes de cette espèce ont été étudiés par RIORDAN (13) qui distingue trois paires de chromosomes, dont deux grandes paires et une paire plus courte. RIORDAN 
n'a pas différenciế les hétérosomes des autosomes.

\section{Sous-genre Glossina s. str. (groupe mor- sitans)}

Toutes les espèces de ce sous-genre ont fait l'objet de recherches cytogénétiques. Les études les plus complètes sont celles de SOUTHERN, en ce qui concerne G.m. morsitans et $G$. austeni. Nous avons, avant les travaux de SOUTHERN, donné une première description du caryotype de ces deux espèces, et nous les avons réexaminés par la suite, à peu près en même temps que cet auteur.

a) Glossina morsitans morsitans Westwood, 1850; MACHADO, 1970.

La garniture chromosomique de cette espèce comprend deux grandes paires d'autosomes (chromosomes $\mathrm{L}_{1}$ et $\mathrm{L}_{2}$ ) plus, en général, quatre petits chromosomes, que SOUTHERN considèrent comme monovalents, et deux chromosomes sexuels (fig. 16 à 23).

Les autosomes $\mathrm{L}_{1}$ sont les plus grands. Ils sont submétacentriques, avec deux bras de longueurs inégales. On peut noter la présence d'une constriction secondaire sur le bras le plus long.

Les autosomes $\mathrm{L}_{2}$ sont plus courts. Les différences de longueur entre les deux bras sont moins accentuées que chez les autosomes $L_{1}$. On n'y décèle pas de constriction secondaire.

Les petits chromosomes sont, dans la plupart des cas, au nombre de quatre. Cependant, chez un certain nombre d'individus, on peut trouver un ou des petits chromosomes en plus, ou en moins. Suivant la terminologie anglosaxonne, nous dénommerons ces petits chromosomes: $S_{\mathfrak{k}}, S_{\mathrm{b}}(\mathrm{S}=$ small). SOUTHERN pense que ces petits chromosomes sont monovalents et ne peuvent être associés par paire. On peut, cependant, nous semble-t-il, distinguer une paire de chromosomes $S_{a}$, de 3 à $4 \mu$ de longueur environ, et une paire de chromosomes $S_{\mathrm{b}}$, plus courts, de 2,3 à $2,5 \mu$ de longueur (mensurations effectuées sur les chromosomes en méiose, à la prophase II). La région centromérique de ces chromosomes est située en position tout à fait terminale.

Les chromosomes sexuels comprennent un chromosome $\mathrm{X}$ et un chromosome $\mathrm{Y}$. La femelle est homogamétique du type $\mathrm{XX}$. Le mâle est hétérogamétique, du type XY.

Le chromosome $\mathrm{X}$ est aussi long, ou légèrement plus long, que l'autosome $\mathrm{L}_{1}$. Il est métacentrique, avec deux bras de longueurs à peu près égales.

Le chromosome $\mathrm{Y}$ est plus petit que l'autosome $\mathrm{L}_{2}$. Il se caractérise par la position $\mathrm{du}$ centromère, situé près d'une extrémité et le divisant en deux bras de longueurs très inégales (fig. 21 et 22).

La formule chromosomique de cette espèce peut en conséquence s'écrire comme suit :

$$
\begin{gathered}
2 \mathrm{n}=2 \mathrm{~L}_{1}+2 \mathrm{~L}_{2}+4 \\
( \pm 1 \text { ou } 2) \mathrm{S}+\mathrm{XX} \text { (ou } \mathrm{XY} \text { ) }
\end{gathered}
$$

\section{b) Glossina austeni Newstead, 1912.}

Cette espèce possède deux grandes paires d'autosomes (paires $\mathrm{L}_{1}$ et $\mathrm{L}_{2}$ ), huit petits chromosomes (chromosomes S) et deux chromosomes sexuels (fig. 24 à 32).

L'autosome $\mathrm{L}_{1}$ est le plus grand des chromosomes. Il est submétacentrique. On peut noter la présence d'une constriction secondaire sur le plus long des deux bras.

L'autosome $\mathrm{L}_{2}$ est plus court (environ d'un tiers) que le $\mathrm{L}_{1}$; il est nettement métacentrique.

Les petits chromosomes $\mathrm{S}$ sont, le plus souvent, au nombre de 8. Dans certaines cellules, cependant, nous avons dénombré 10 et quelquefois 12 chromosomes $S$. Leurs dimensions sont comprises entre 2,8 et $4,7 \mu$ et ils peuvent généralement, mais non régulièrement, être associés par paire. Le centromère paraît le plus souvent être en position terminale.

SOUTHERN trouve également des variations considérables, dans le nombre de ces chromosomes, d'un individu à l'autre, ce nombre pouvant aller de 8 à 12 , avec une majorité d'individus possédant un jeu de 10 chromosomes S. Tous les chromosomes S sont télocentriques (centromère terminal), hétérochromatiques, et probablement univallents.

Les hétérosomes comprennent, comme dans les autres espèces, un chromosome $X$ et un chromosome $\mathrm{Y}$. La femelle est homogamétique $(\mathrm{XX})$ et le mále hétérogamétique (XY).

Les chromosomes $\mathrm{X}$ sont à peu près de même longeur que les autosomes $\mathrm{L}_{1}$, mais ils 
sont métacentriques et ne possèdent pas de constriction secondaire.

Le chromosome $\mathrm{Y}$ est un peu plus court que le chromosome $\mathrm{X}$, mais plus long que l'autosome $L_{\mathfrak{g}}$, et la position de son centromère permet de distinguer deux bras de longueurs inégales, cette différence étant cependant beaucoup moins accusée que dans le cas du chromosome $\mathrm{Y}$ de G.m. morsitans.

Le caryotype de $G$. austeni diffère donc de celui de G.m. morsitans, d'une part, par un nombre plus élevé de chromosomes $S$, et d'autre part, par les dimensions du chromosome $\mathrm{Y}$ et la position de son centromère.

La formule chromosomique de $G$. austeni peut s'écrire de la façon suivante:

$$
\begin{gathered}
2 \mathrm{n}=2 \mathrm{~L}_{1}+2 \mathrm{~L}_{2}+10 \\
( \pm 2) \mathrm{S}+\mathrm{XX} \text { (ou } \mathrm{XY})
\end{gathered}
$$

c) G. morsitans centralis MACHADO, 1970.

Cette espèce a été étudiée par MAUDLIN (11), sous le nom de G.m. morsitans Westwood, puis, tout récemment, par SOUTHERN (sous presse).

MAUDLIN trouve 8 chromosomes chez cette espèce, dont trois grandes paires et une petite paire. Une des grandes paires est submétacentrique, toutes les autres étant métacentriques. MAUDLIN ne différencie pas les hétérosomes des autosomes.

SOUTHERN, dans une communication présentée, en 1972 , au $13^{\mathrm{e}}$ séminaire sur les trypanosomoses de Londres, mentionne que G.m. centralis possède deux paires de grands autosomes submétacentriques $\left(\mathrm{L}_{1}\right.$ et $\left.\mathrm{L}_{2}\right)$ plus un petit chromosome métacentrique $\left(\mathrm{M}_{3}\right)$, non présent chez les autres espèces de ce sous-genre, et deux chromosomes sexuels (XX chez la femelle, XY chez le mâle). Le chromosome X est semblable à l'autosome $\mathbf{L}_{1}$. Le chromosome $\mathrm{Y}$ est plus court et submétacentrique. Il est semblable au chromosome Y de $G$. austeni.

Il ne semble pas que G.m. centralis possède de petits chromosomes $\mathrm{S}$. Sa formule serait donc :

$2 \mathrm{n}=2 \mathrm{~L}_{1}+2 \mathrm{~L}_{2}+2 \mathrm{M}_{3}+\mathrm{XX}$ (ou XY)

d) G. morsitans submorsitans Newstead, 1910.

Dans cette même communication, SOUTHERN précise que G.m. submorsitans pos- sède deux paires de grands autosomes submétacentriques $\left(\mathrm{L}_{1}\right.$ et $\left.\mathrm{L}_{2}\right)$, un jeu de petits chromosomes télocentriques (S) qui varie de 5 à 7 , et deux chromosomes sexuels (XX chez la femelle, XY chez le mâle). Ces hétérosomes seraient semblables à ceux de G.m. centralis et de $G$. austeni.

La formule chromosomique de G.m. submorsitans serait donc:

$$
\begin{gathered}
2 \mathrm{n}=2 \mathrm{~L}_{1}+2 \mathrm{~L}_{2}+6 \\
( \pm 1) \mathrm{S}+\mathrm{XX} \text { (ou XY) }
\end{gathered}
$$

e) G. longipalpis Wiedmann, 1830

BALDRY (1) donne une description sommaire du caryotype de cette espèce qui possèderait deux grandes paires, une paire moyenne et une petite paire de chromosomes. Les grands et les moyens chromosomes seraient métacentriques; les petits chromosomes (chromosomes $\mathrm{S}$ ?) seraient acrocentriques. BALDRY n'a pas différencié les chromosomes sexuels.

f) G. pallidipes Austen, 1903.

HULLEY (4) et MAUDLIN (11) ont successivement décrit le caryotype de cette espèce. Selon HULLEY, G. pallidipes possède 8 chromosomes, soit deux grandes paires, une paire moyenne et une petite paire (chromosome $S$ ?). Les six premiers chromosomes sont métacentriques. HULLEY a noté, chez certains individus, la perte d'un petit chromosome; il présente en outre une figure où l'un des grands chromosomes est sub-acrocentrique. Nous pensons que ce chromosome pourrait être un chromosome Y.

MAUDLIN a examiné des pupes de $G$. pallidipes provenant de deux localités ditférentes (Kariba en Rhodésie et Lugula en Uganda). Chez les individus provenant de Rhodésie, il trouve 8 chromosomes (deux paires et une paire moyenne, métacentriques; une petite paire, acrocentrique), ce qui correspond à la description de HULLEY. Par contre, les individus provenant de l'Uganda ne possèdent pas de petits chromosomes. Les individus issus de croisements entre mâles d'Uganda et femelles de Rhodésie ne possèdent également que 6 chromosomes ( 2 grandes paires et 1 paire moyenne, métacentriques), comme leur parent mâle.

g) G. swynnertoni Austen, 1923.

MAUDLIN (11) indique que cette espèce 
aurait huit chromosomes, répartis en quatre grandes paires, toutes métacentriques. MAUDLIN constate que ce caryotype est très proche de celui de G.m. centralis, bien que les chromosomes de la quatrième paire soient, semblet-il, plus longs que les chromosomes $\mathrm{M}_{3}$ de cette dernière espèce.

\section{Sous-genre Austenina (groupe fusca)}

Deux espèces seulement ont été étudiées, l'une, G. brevipalpis, par MAUDLIN (11), l'autre, G. fusca congolensis, par ITARD (9).

a) G. fusca congolensis Newstead et Evans, 1921.

Cette espèce possède 22 chromosomes (fig. 33 à 37), dont 10 paires d'autosomes et une paire de chromosomes sexuels.

Les autosomes peuvent être classés en trois grandes paires, métacentriques $\left(\mathrm{L}_{1}, \mathrm{~L}_{2}, \mathrm{~L}_{3}\right)$; six paires un peu moins longues, submétacentriques $\left(\mathrm{L}_{4}\right.$ à $\left.\mathrm{L}_{8}\right)$; une petite paire, métacentrique $\left(\mathrm{M}_{10}\right)$.

Les chromosomes sexuels sont métacentriques. Le chromosome $X$ a sensiblement les mêmes dimensions que les autosomes $\mathrm{M}_{10}$. Le chromosome $\mathrm{Y}$ est le plus petit de tous les chromosomes. La femelle possède deux $\mathrm{X}$ et le mâle un $X$ et un $Y$.

b) G. brevipalpis Newstead, 1910.

Cette espèce a 16 chromosomes (huit paires), tous métacentriques, mais MAUDLIN ne donne pas de plus amples précisions sur les dimensions respectives de ces chromosomes et les rapports de longueur de leurs bras.

\section{CHROMOSOMES POLYTENIQUES}

Les chromosomes polyteniques de G.m. morsitans ont été étudiés par SOUTHERN (17). Seuls les autosomes $\mathrm{L}_{1}$ et $\mathrm{L}_{2}$ et le chromosome $\mathrm{X}$ forment des éléments polyténiques. Les chromosomes $S$ et le chromosome $\mathrm{Y}$ (chromosomes hétérochromatiques), ne sont pas décelables dans les noyaux des cellules géantes étudiées (cellules trichogènes et tormogènes des machrochètes thoraciques de pupes de 17 jours).

Il n'y a pas de chromocentre commun et, le plus souvent, les deux bras de chaque élément polyténique sont séparés au centromère. En comparant les longueurs relatives des bras des chromosomes en mitose et en méiose avec ceux des chromosomes polyténiques et en étudiant les chromosomes polyténiques d'individus porteurs de translocations produites par irradiation gamma, SOUTHERN a pu dresser la carte des chromosomes géants chez cette espèce et identifier plus de 600 bandes.

\section{CONCLUSIONS}

L'étude systématique de la cytogénétique des glossines a commencé il y a seulement six ans. Des progrès considérables ont été réalisés dans ce court laps de temps, mais il apparaît évident que nos connaissances sont encore très limitées. Sur les trente espèces ou sous-espèces que comporte le genre Glossina, douze seulement ont fait l'objet de recherches plus ou moins complètes. Des descriptions assez précises ont été données pour les caryotypes de deux espèces du sous-genre Nemorhina, quatre especes du sous-genre Glossina et une espèce du sousgenre Austenina. Les caryotypes des cinq autres espèces doivent être réexaminés, en particulier pour la différenciation des chromosomes sexuels.

Malgré ces importantes lacunes, il semble se dégager une certaine homogénéité à l'intérieur d'un même sous-genre. Dans le sous-genre Nemorhina, les espèces étudiées ne possèdent que 6 chromosomes (4 grands autosomes et 2 chromosomes sexuels). Chez les espèces du sous-genre Glossina, on trouve 4 grands autosomes et 2 chromosomes sexuels, plus un nombre variable de petits chromosomes. Les espèces du sous-genre Austenina paraissent posséder un nombre élevé de grands autosomes (de 14 à 20) plus deux chromosomes sexuels.

D'autre part, les recherches effectuées par SOUTHERN chez G.m. morsitans et G. austeni semblent indiquer que la méiose, chez le mâle, est achiasmatique chez $G$. austeni, alors que des chiasmas peuvent se produire, mais à une très faible fréquence, chez G.m. morsitans.

Le fait que l'on soit maintenant capable d'identifier, au moins chez quelques espèces, les chromosomes avec certitude, et, dans un cas tout au moins, d'avoir pu dresser la carte des chromosomes polyténiques, ouvre la voie à tout un champ de recherches, non seulement 
dans le domaine de la systématique, pour la différenciation des sous-espèces, mais également dans le domaine de la génétique appli- quée à la lutte autocide, avec la possibilité d'étudier les chromosomes d'hybrides, d'individus porteurs de translocations ou de mutants.

\title{
SUMMARY
}

\section{Review of present knowledges of cytogenetic of Glossina (Diptera)}

The karyotypes of twelve species of Glossina have been described by various authors in the last six years. Among these species, however, only seven species have sufficiently been studied: two species of the palpalis group ( $G$. fuscipes fuscipes and $G$, tachinoides), four species of the morsitans group (G.m. morsitans, G.m. centralis, G.m. submorsitans and $G$. austeni) and one species of the fusca group ( $G$. fusca congolensis).

Moreover, Polytene chromosomes maps have been constructed for one species (G.m. morsitans).

\section{RESUMEN}

\section{Revista de los conocimientos actuales sobre la citogenetica de las glosinas (Diptera)}

\begin{abstract}
Varios autores describieron los cariotipos de doce especies de glosinas durante los seis ultimos años. Sin embargo se estudiaron relativamente bien solo siete especies de ellas : dos especies del grupo palpalis ( $G$. fuscipes y $G$. tachinoides), cuatro especies del grupo morsitans (G.m. morsitans, G.m. centralis, G.m. submorsitans y $G$. austeni) y una especie del grupo fusca (G. fusca congolensis).

Se pudo, ademas, establecer la mapa de los cromosomos politenicos de una especie (G.m. morsitans).
\end{abstract}

\section{BIBLIOGRAPHIE}

1. BALDRY (D. A. T.). A rapid staining technique for demonstrating the mitotic chromosomes of Glossina spp. - Criação de mosca tse-tse em laboratorio. $1^{\text {er }}$ Symposium intern. Lisbonne 1970: 349-354.

2. BURCHARD (R. P.) et BALDRY (D. A. T.). Polytene chromosomes of Glossina palpals R.-D. (Diptera Muscidae). I. The preliminary demonstration. Proc. R. ent. Soc. Lond. (A). 1970, 45 (10-12): 182-183.

3. CRAIG CAMERON (T. A.), SOUTHERN (D. I.) et PELL (P. E.). Chromosomes ultrastructure at meiosis in $G$. austeni and $G$. morsitans morsitans. Trans. R. Soc. trop. Med. Hyg., 13e Seminar on Trypanosomiasis (sous presse).

4. HULLEY (P. E.). Mitotic chromosomes of Glossina pallidipes Austen. Nature, 1968, 217 (5132): 977-979.

5. ITARD (J.). Chromosomes de Glossines (DipteraMuscidae). C.R. Acad. Sci. Paris, 1966, 263 série D (19) : 1395-1397.

6. ITARD (J.). Observations sur les caryotypes de quatre espèces de glossines. 1.S.C.T.R. - 12e Réunion - Bangui, R.C.A. Novembre 1968.

7. ITARD (J.). Les caryotypes de six espèces de Glossines. Criação de mosca tse-tse em laboratorio. $1^{\text {er }}$ Symposium intern. Lisbonne, 1970: 361-367.

8. ITARD (J.). L'appareil reproducteur mâle des glossines (Diptera-Muscidae). Les étapes de sa formation chez la pupe. La spermatogénèse. Rev. Elev. Méd. vét. Pays trop., 1970, 23 (1) : 57-81.

9. ITARD (J.). Chromosomes de Glossina fusca congolensis Newstead et Evans, 1921 (Diptera-Muscidae) - C.R. Acad. Sci, Paris, 1971, 272 série D (20): 2561-2564.
10. MAUDLIN (I.). Chromosomes of tsetse fly Glossina fuscipes fuscipes Newstead. E.A.T.R.O. Annual report, 1968: 64-66.

11. MAUDLIN (I.). Preliminary studies on the karyotypes of five species of Glossina. Parasitology, 1970, 61: 71-74.

12. PELL (P.E.), SOUTHERN (D. I.) et CRAIGCAMERON (T.A.). Comparative cytogenetics of the morsitans group. Trans. $R$. Soc. trop. Med. Hyg., $13^{\mathrm{e}}$ Seminar on Trypanosomiasis (sous presse).

13. RIORDAN (K.). Chromosomes of the tsetse fly, Glossina palpalis. Parasitology, 1968, 58 (4): 835-838.

14. RIORDAN (K.). Polytene chromosomes of Glossina palpalis R.-D. (Diptera: Muscidae). II - Their improved demonstration. Proc. R. ent. Soc, Lond. (A), 1970, 45 (10-12): 184-186.

15. SOUTHERN (D. I.), CRAIG-CAMERON (T. A.) et PELL (P.E.). The meiotic sequence in Glossina morsitans morsitans. Trans. R. Soc. trop. Med. Hyg., 1972, 66 (1) : 145-149.

16. SOUTHERN (D.I.), CRAIG-CAMERON (T. A.) et PELL (P.E.). A critical chromosome analysis of Glossina austeni Newst. (Dipt., Glossinidae). Bull. ent. Res., 1972, 62 (2) : 195-198.

17. SOUTHERN (D. I.), PELL (P.E.) et CRAIGCAMERON (T.A.). Polytene chromosomes of the Tsetse fly Glossina morsitans morsitans. Chromosoma (Berl.), 1973, 40 : 107-120.

18. VANDERPLANK (F.L.). Experiments in cross breeding tsetse flies (Glossina species). Ann. trop. Med. Parasit., 1948, 42 : 131-52.

19. WRIGHT (J.W.) et PAL (R.). Genetics of insect vectors of disease. Amsterdam, Elsevier publ. Co., 1967, 794 p. 


\section{GLOSSINA FUSCIPES FUSCIPES}

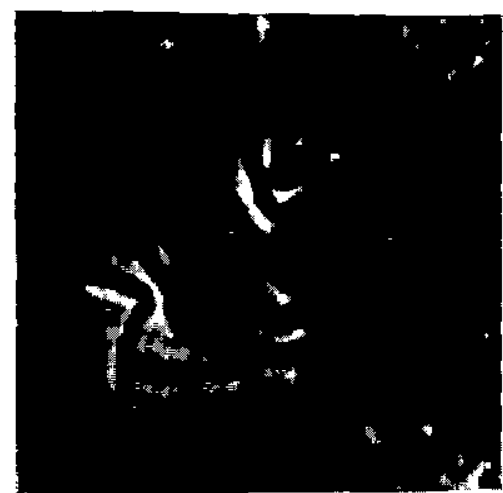

Fig. 1. - G. fuscipes fuscipes femelle. Métaphase dans une cellule nerveuse (pupe de 8 jours).

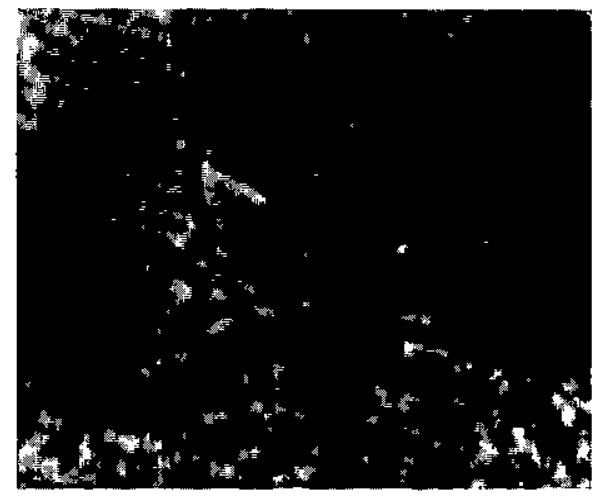

Fig. 3. - G. fuscipes furcipes mâle. Métaphase dans une cellule nerveuse (pupe de 8 jours).

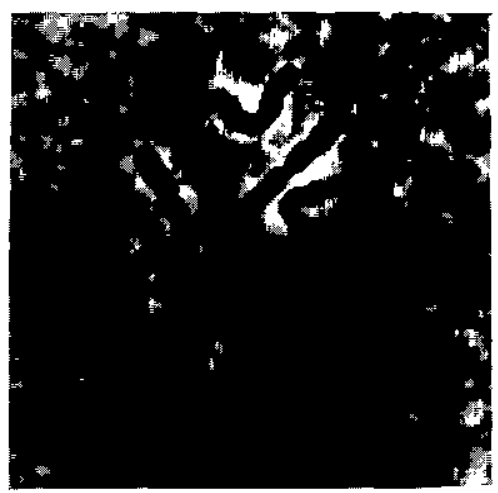

Fig. 2. - G. fuscipes fuscipes mâle. Métaphase dans une cellule nerveuse (pupe de 8 jours).

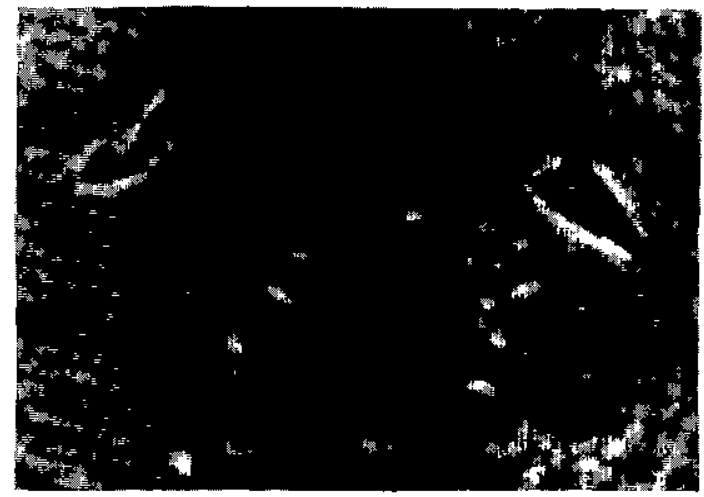

Fig. 4. - G. fuscipes fuscipes (spermatocyte d'une pupe de 8 jours). Anaphase I.

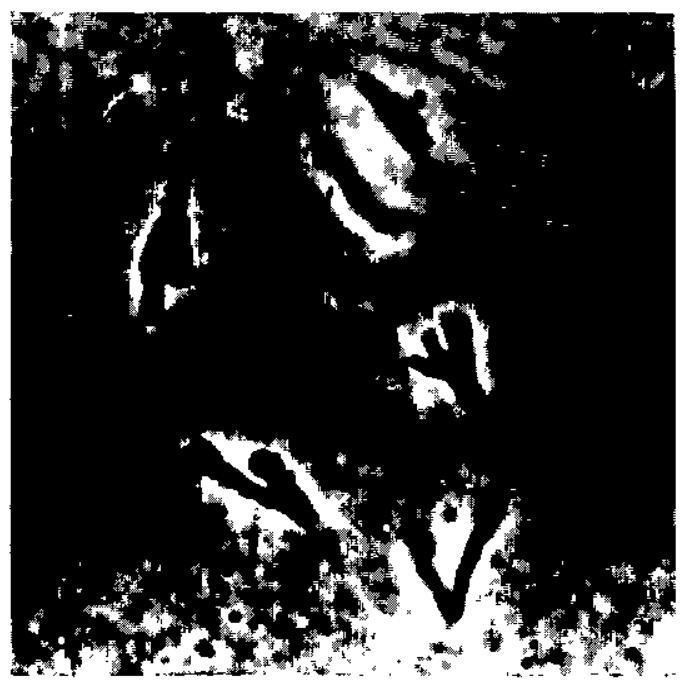

Fig. 5. - G. fuscipes fuscipes (spermatocyte d'une pupe de 8 jours). Fin de l'anaphase $I$. 


\section{GLOSSINA FUSCIPES FUSCIPES (suite)}

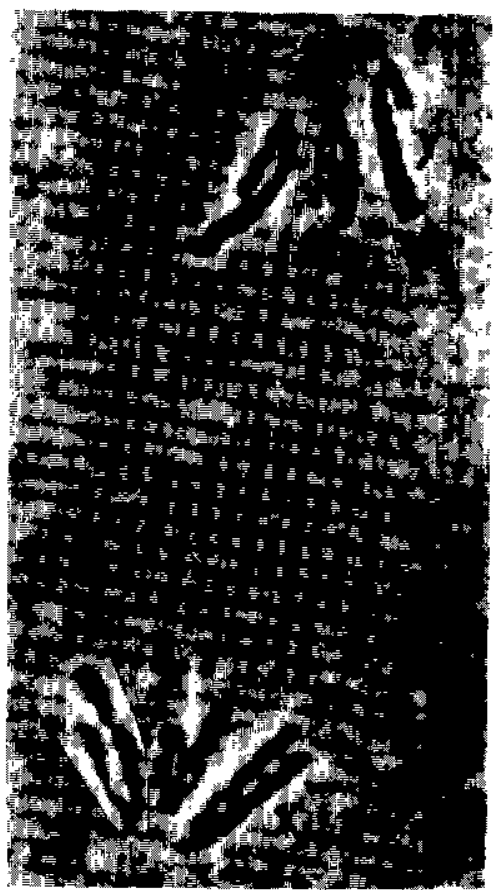

Fig, 6. $\rightarrow G$. fuscipes fuscipes (spermatocyte d'une pupe de 8 jours). Début de la télophase 1 .

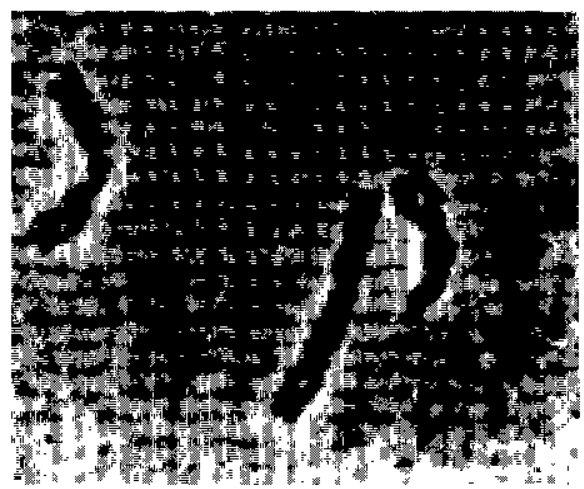

Fig. 7. - G. fuscipes fuscipes. Prophase II. Un chromosome $\mathrm{X}$.

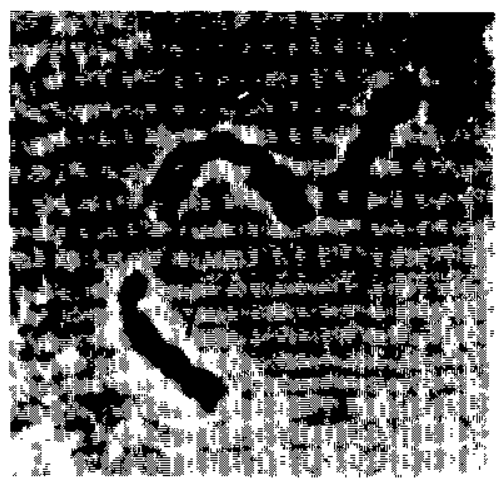

Fig. 8. - G. fuscipes fuscipes. Prophase II. Un chromosome $Y$.

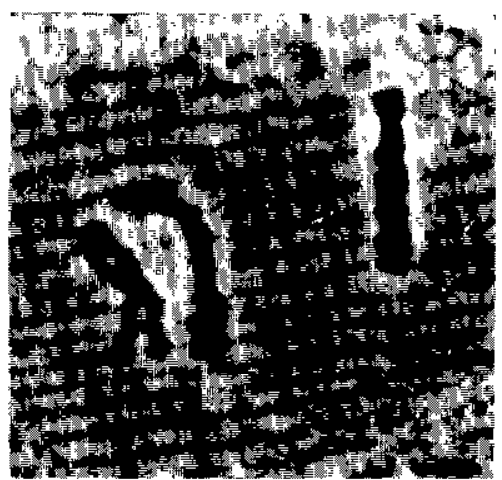

Fig. 9. - G. fuscipes fuscipes. Prophase II. Un chromosome $\mathrm{Y}$. 


\section{GLOSSINA TACHINOIDES}

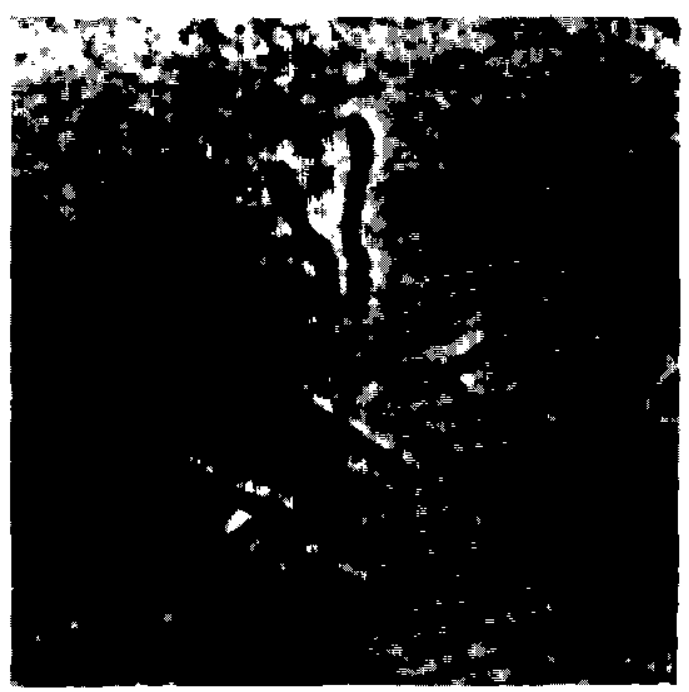

Fig. 10. - G. tachinoides femelle. Métaphase dans une cellule nerveuse (pupe de 7 jours).
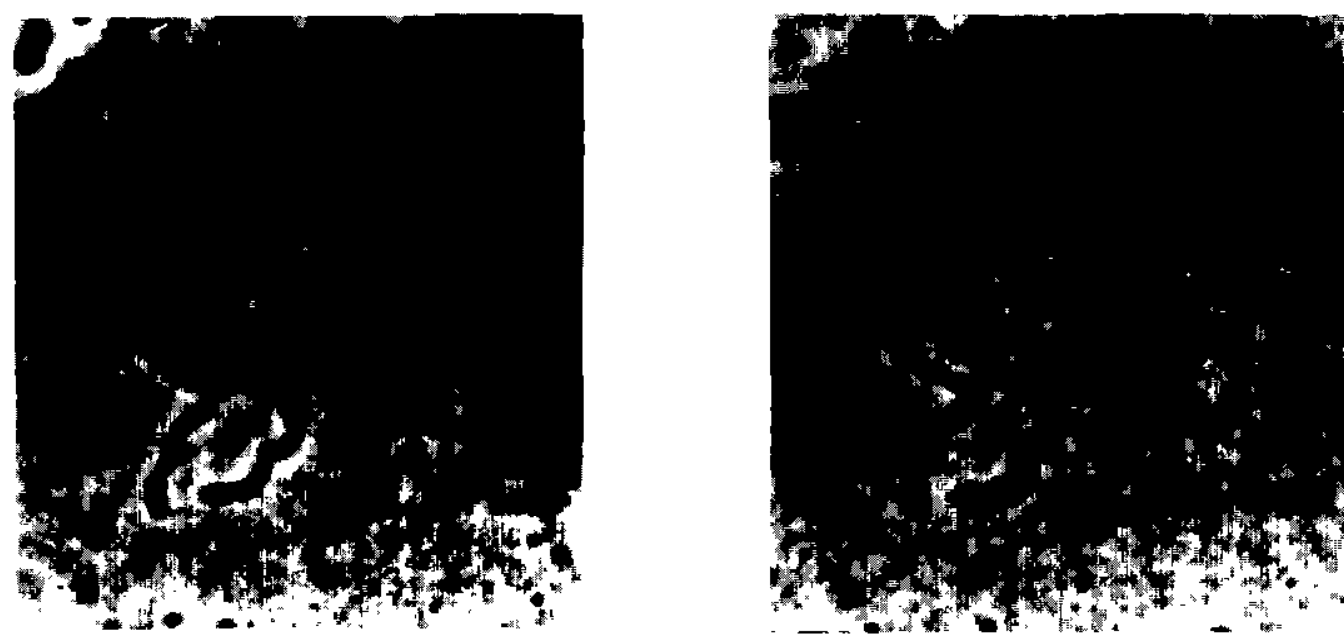

Fig. 11 et 12 . $-G$. tachinoides mâle. Métaphase dans une cellule nerveuse (pupe de 9 jours). Le chromosome Y n'est pas dans le même plan que les autres chromosomes. 


\section{GLOSSINA TACHINOIDES (suite)}

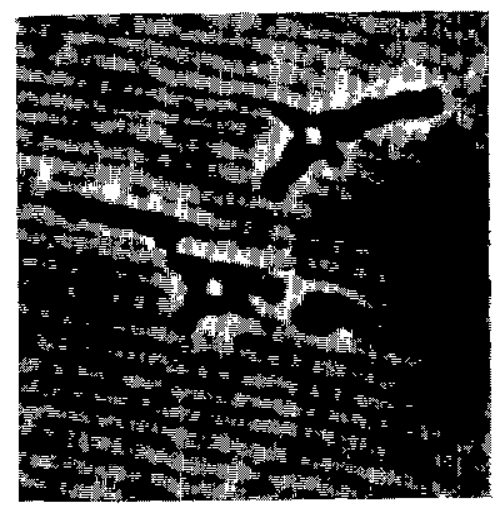

Fig. 13. - G. tachinoides (spermatocyte d'une pupe de 7 jours). Fin de la métaphase 1. Début de l'anaphase I.

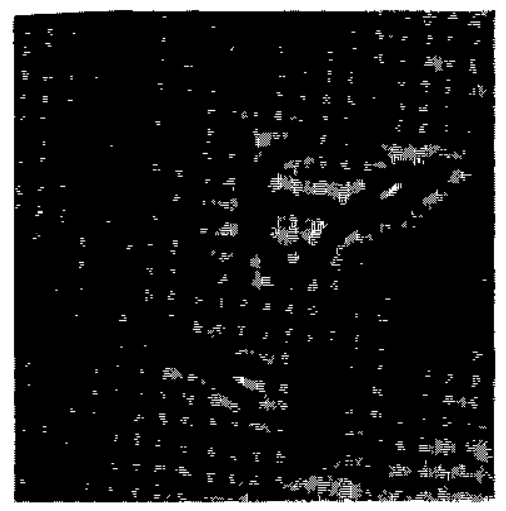

Fig. 14. - G. tachinoides. Prophase II. Un chromosome $\mathrm{X}$.

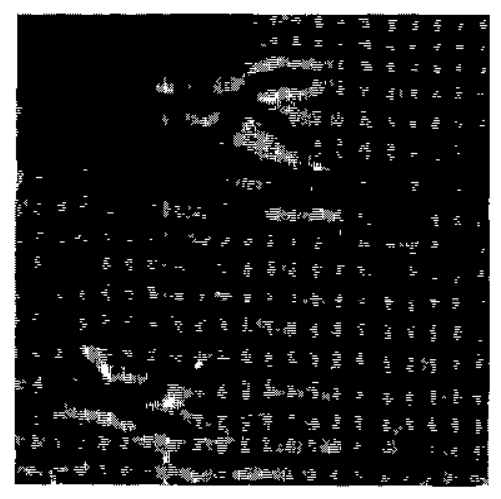

Fig. 15. - G. tachinoides. Prophase II. Un chromosome $\mathrm{Y}$. 


\section{GLOSSINA M. MORSITANS}
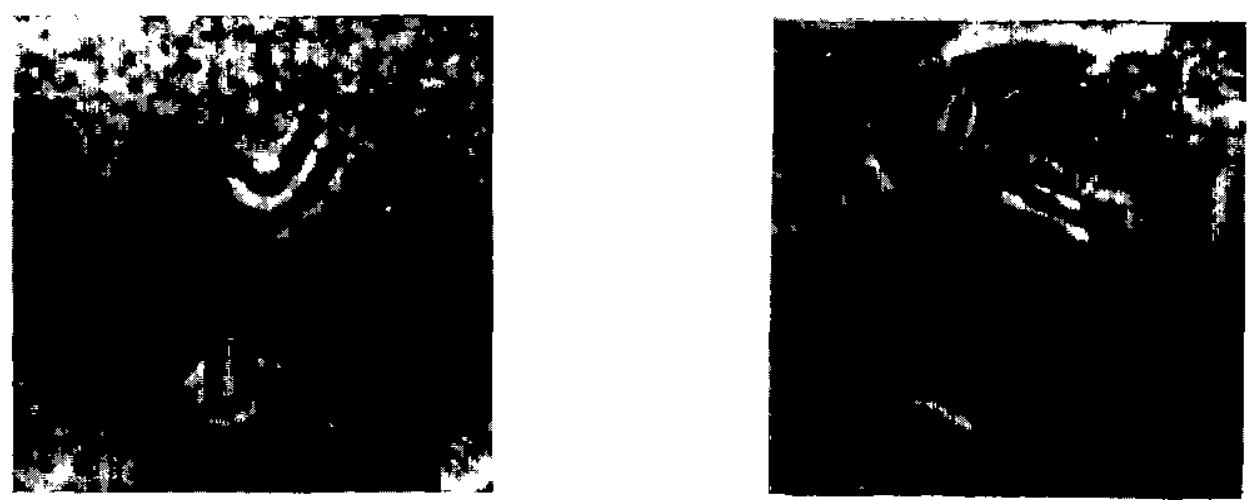

Fig. 16. - G.m. morsitans mâle. Métaphase dans une cellule nerveuse (pupe de 8 jours). 3 chromosomes $S$.

Fig. 17. - G.m. morsitans mâle. Métaphase dans une cellule nerveuse (pupe de 7 jours). 4 chromosomes $S$.

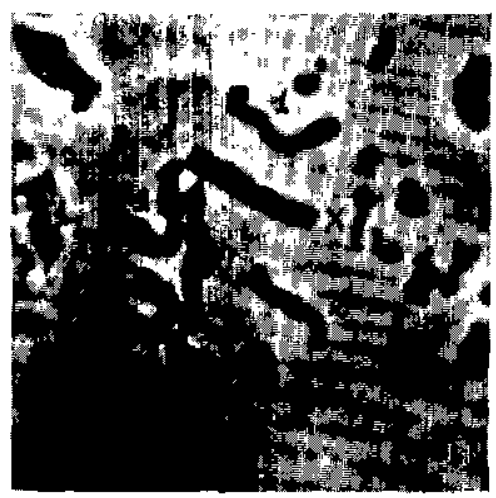

Fig. 18. - G.m. morsitans femelle. Métaphase dans une cellule nerveuse (pupe de 7 jours). 4 chromosomes $\mathrm{S}$.

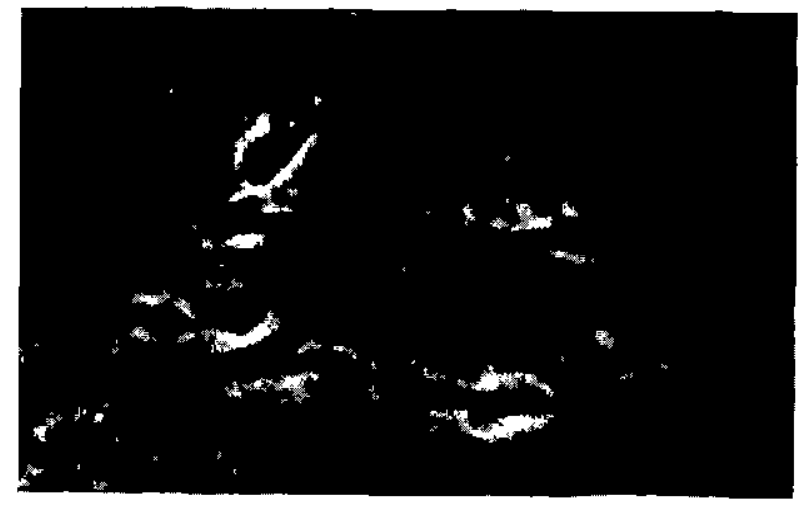

Fig. 19. - G.m. morsitans (spermatocyte d'une pupe de 8 jours). Anaphase I. 5 chromosomes S. 
GLOSSINA M. MORSITANS (suite)

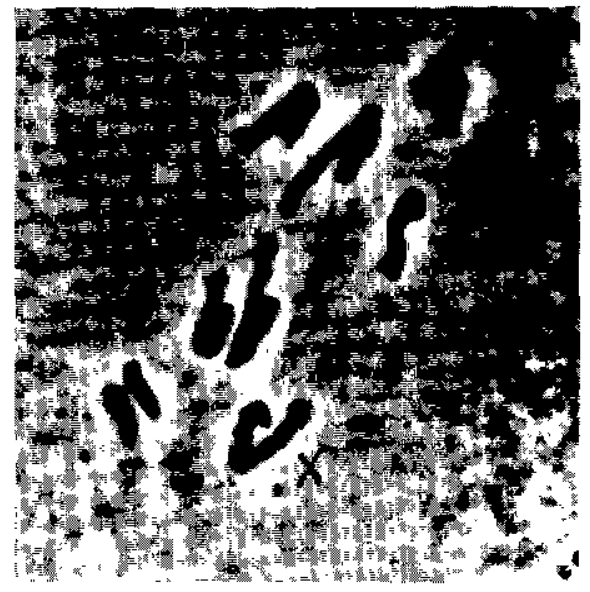

Fig. 20. - G.m. morsitans (spermatocytes d'une pupe de 8 jours). Anaphase I. 4 chromosomes $\mathbf{S}$.

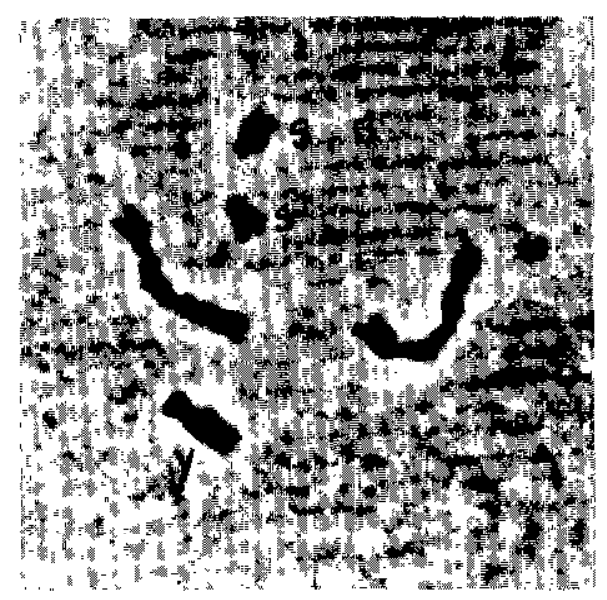

Fig. 21. - G.m. morsitans. Prophase II. Un chromosome $\mathbf{Y}$.

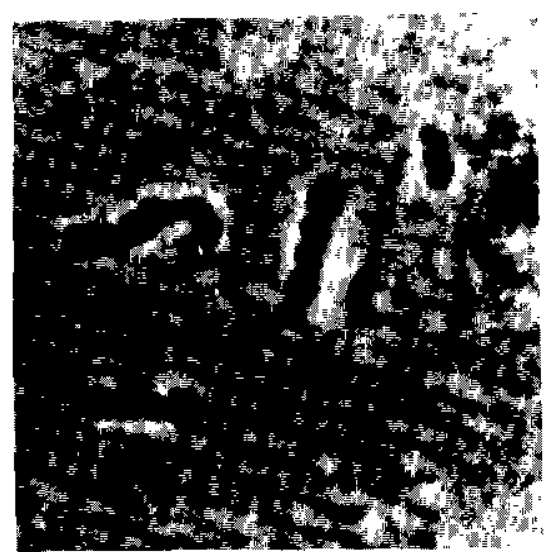

Fig, 22. - G.m. morsitans, Prophase II. Un chromosome $\mathrm{Y}$.

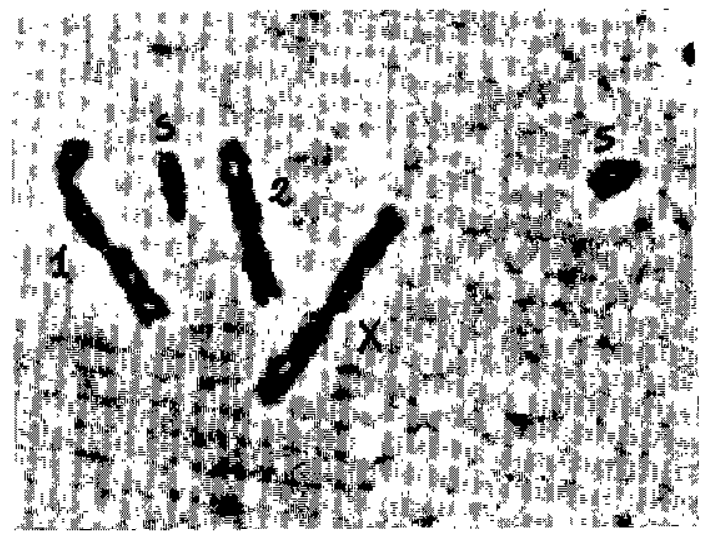

Fig. 23. - G.m. morsitans. Prophase II. Un chromosome $\mathrm{X}$. 


\section{GLOSSINA AUSTENI}

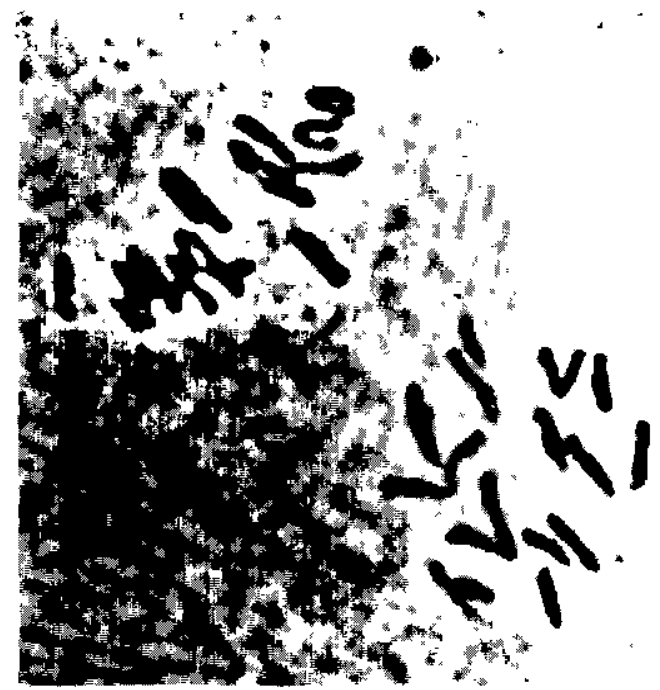

Fig. 24. $-G$. austeni mâle. Anaphase dans une cellule nerveuse (pupe de 7 jours). 8 chromosomes $S$ (2 $\mathrm{n}=14)$.

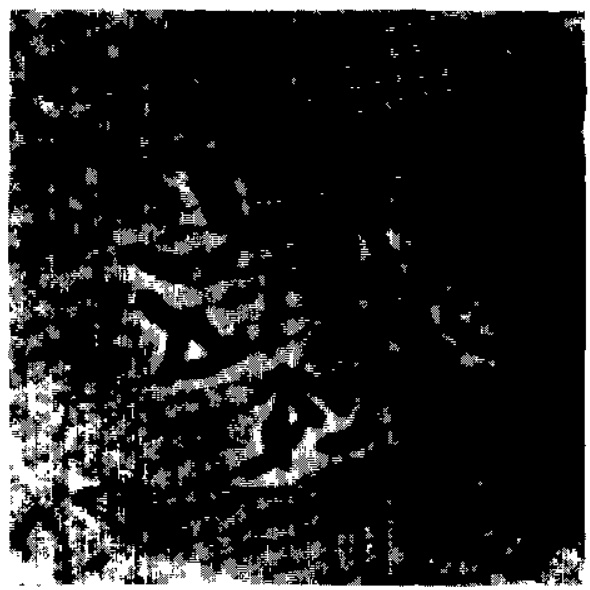

Fig. 26. - G. austeni femelle. Métaphase dans une cellule nerveuse (pupe de 7 jours), 10 chromosomes $\$$ $(2 \mathrm{n}=16)$.

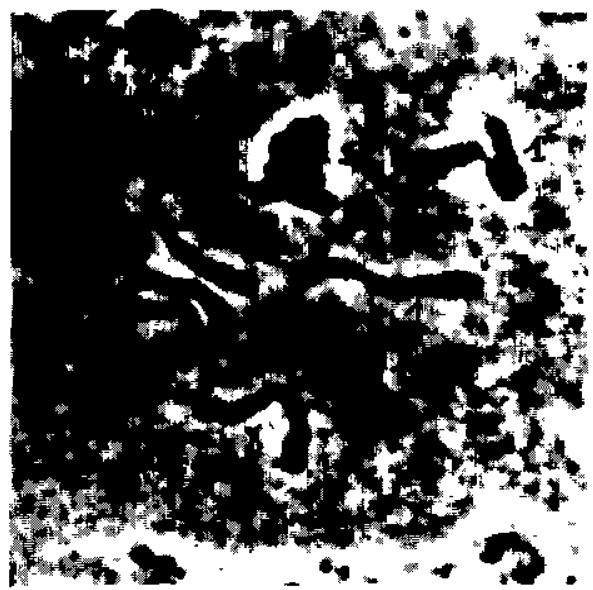

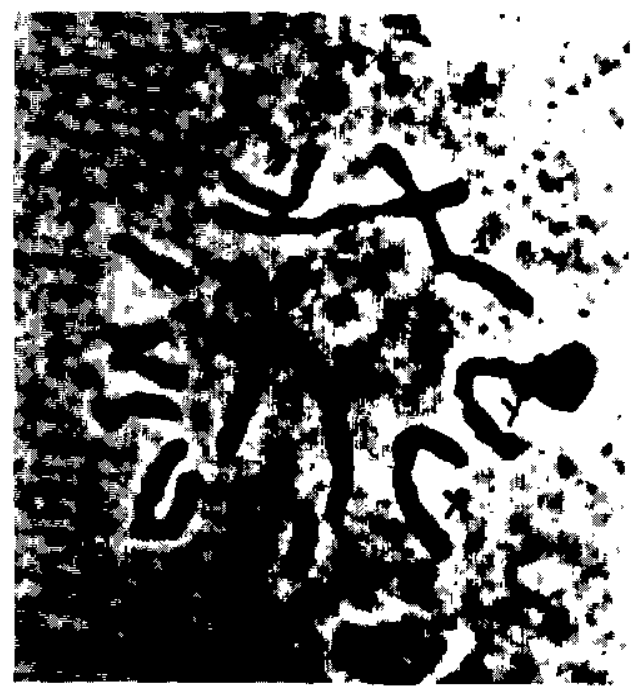

Fig. 25. - G. austeni mâle. Métaphase dans une cellule nerveuse (pupe de 7 jours). 8 chromosomes $S$ $(2 n=14)$.

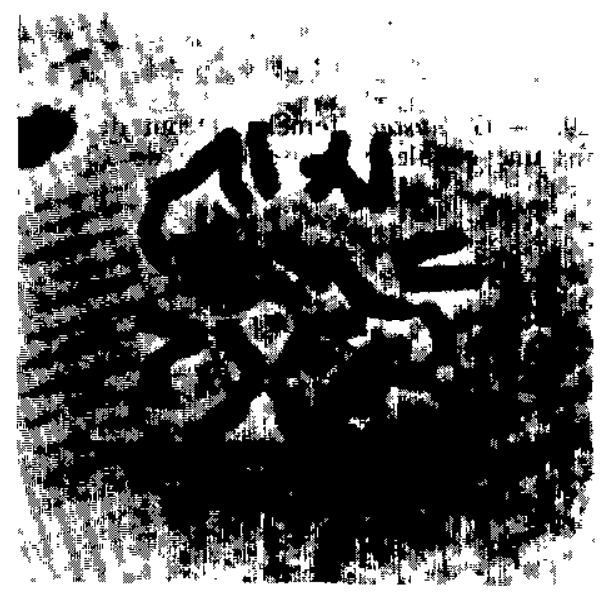

Fig. 27. - G. austeni femelle. Métaphase dans une cellule nerveuse (pupe de 7 jours). 10 chromosomes $S$ (2 $n=16)$.

Fig. 28. - G. austeni femelle. Prophase dans une cellule nerveuse (pupe de 10 jours). Les chromosomes $\mathbf{S}$ ne sont pas dissociés. 
GLOSSINA AUSTENI (suite)

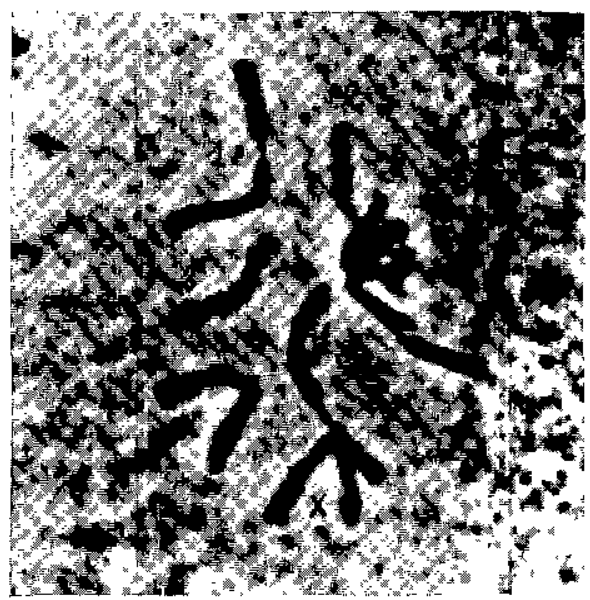

Fig. 29. - G. austeni femelle. Début de métaphase dans une cellule nerveuse (pupe de 10 jours).

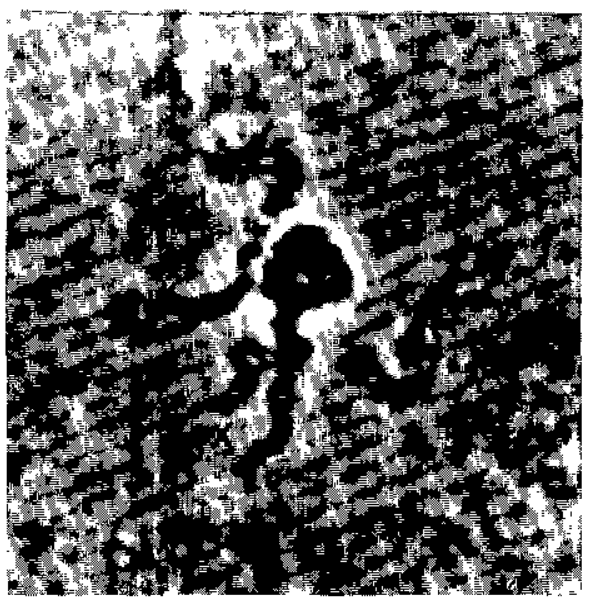

Fig. 30. - G. austeni (spermatocyte d'une pupe de 11 jours). Début de la prophase méiotique.

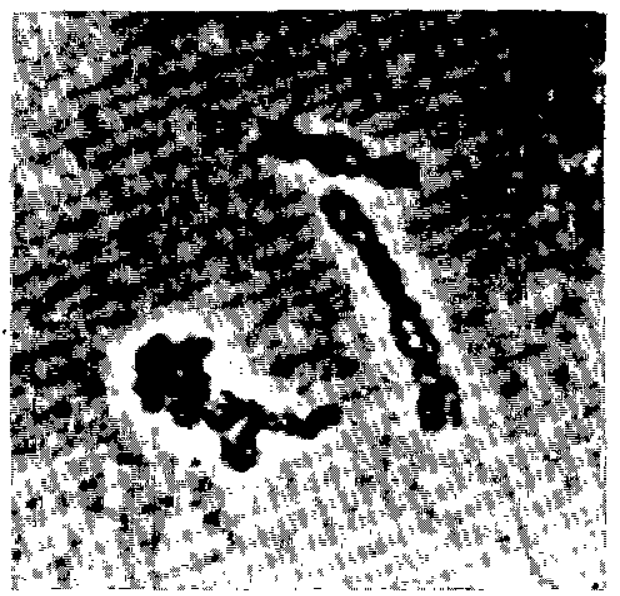

Fig. 31. - G. austeni (spermatocyte dune pupe de 11 jours). Début de la prophase méiotique.

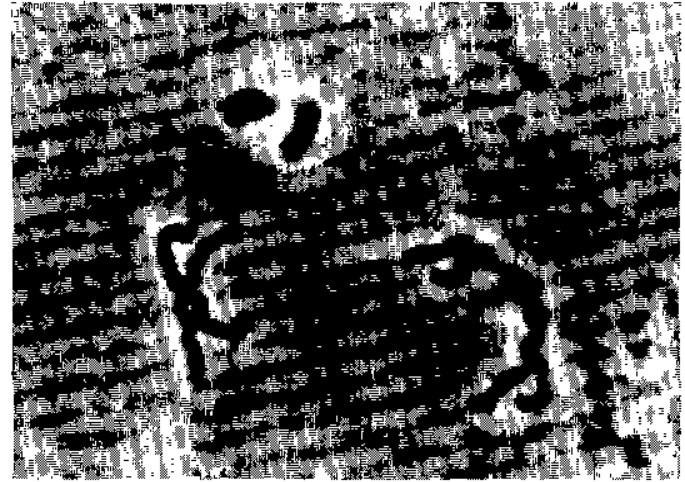

Fig. 32. - G. austeni (spermatocyte d'une pupe de 12 jours). Prophase II. Un chromosome X. 


\section{GLOSSINA FUSCA CONGOLENSIS}

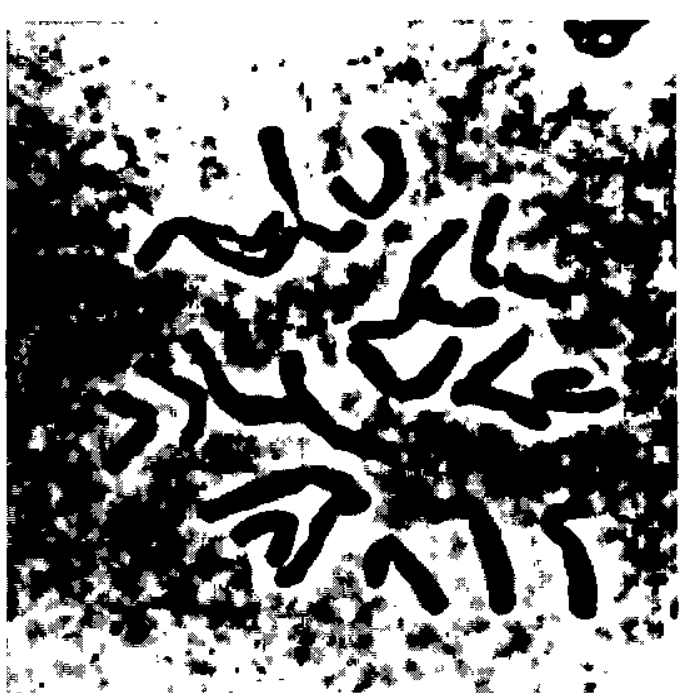

Fig. 33. - G. fusca congolensis femelle. Métaphase dans une cellule nerveuse (pupe de 9 jours). $2 \mathbf{n}=22$.

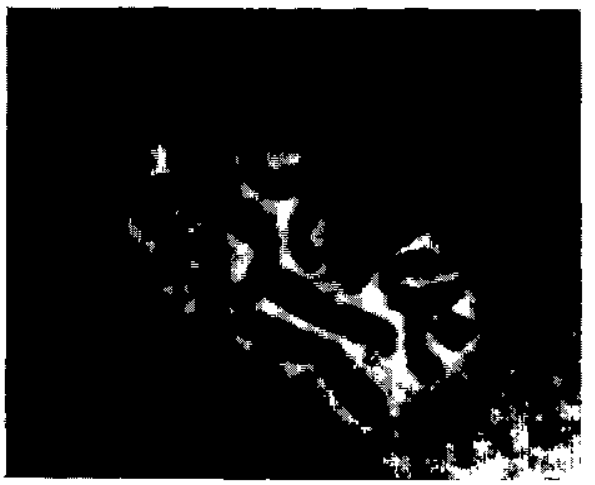

Fig. 35, - G. fusca congolensis (spermatocyte d'une pupe de 8 jours). Métaphase II. Un chromosome $X$.

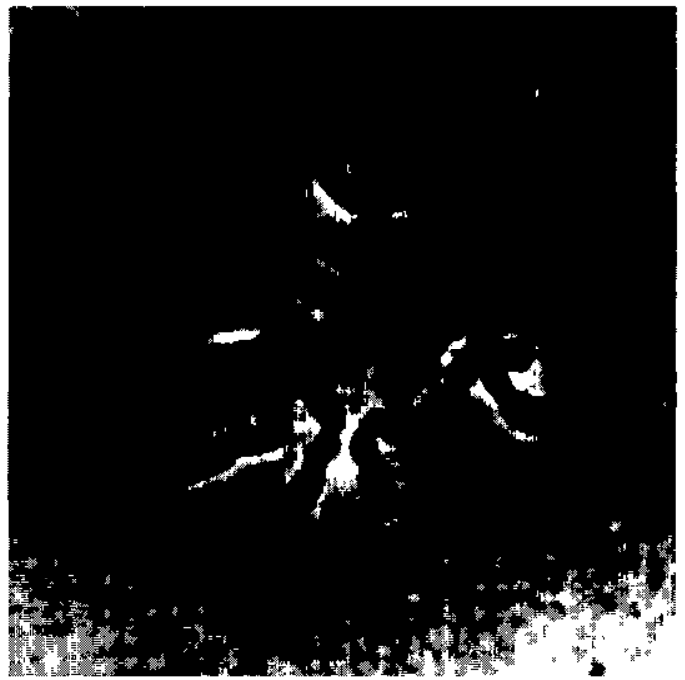

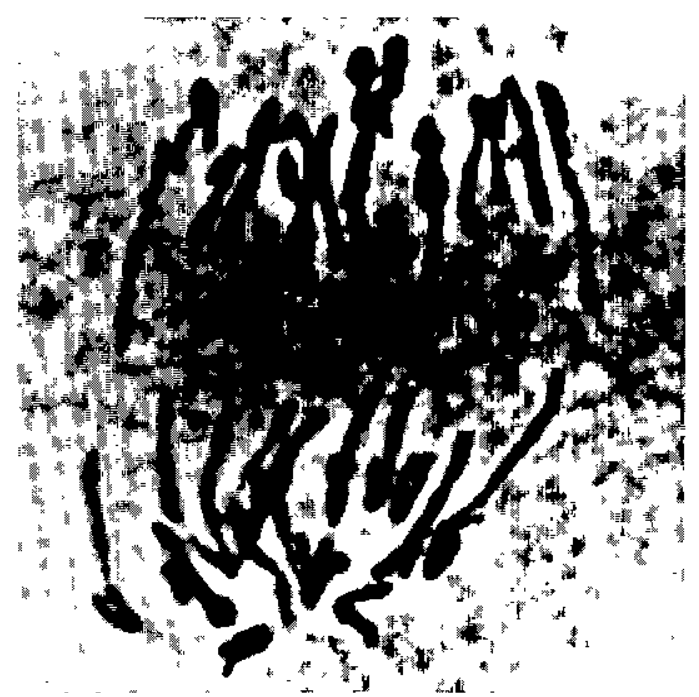

Fig. 34. - G. fusca congolensis femelle. Anaphase dans une cellule nerveuse (pupe de 9 jours).

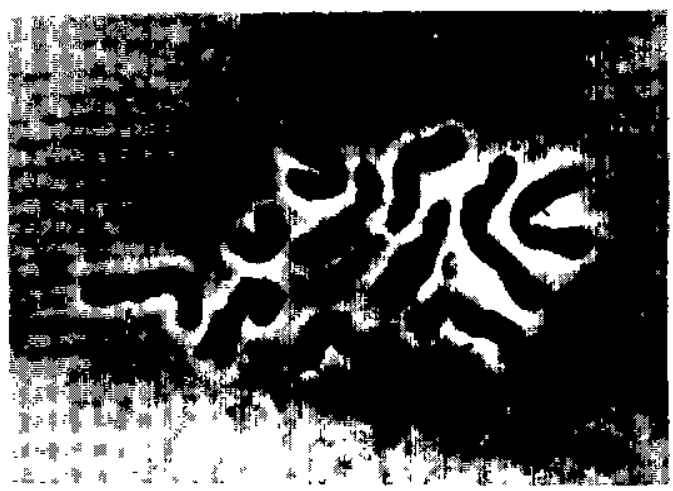

Fig. 36. - G. fusca congolensis (spermatocyte d'une pupe de 8 jours). Métaphase II. Un chromosome $Y$.

Fig. 37. - G. fusca congolensis (spermatocyte d'une pupe de 8 jours). Métaphase II. Un chromosome $Y$ superposé à l'un des bras du chromosome $\mathrm{L}_{2}$. 